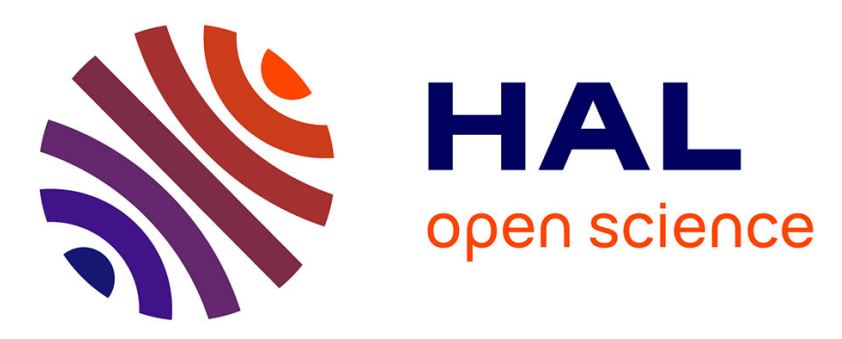

\title{
Non-destructive evaluation of ceramic porosity using terahertz time-domain spectroscopy
}

Davit Hakobyan, Maher Hamdi, Olivier Redon, Anthony Ballestero, Alexis Mayaudon, Laurence Boyer, Olivier Durand, Emmanuel Abraham

\section{To cite this version:}

Davit Hakobyan, Maher Hamdi, Olivier Redon, Anthony Ballestero, Alexis Mayaudon, et al.. Nondestructive evaluation of ceramic porosity using terahertz time-domain spectroscopy. Journal of the European Ceramic Society, 2022, 42 (2), pp.525-533. 10.1016/j.jeurceramsoc.2021.10.026 . hal03525103

\section{HAL Id: hal-03525103 \\ https://hal.science/hal-03525103}

Submitted on 13 Jan 2022

HAL is a multi-disciplinary open access archive for the deposit and dissemination of scientific research documents, whether they are published or not. The documents may come from teaching and research institutions in France or abroad, or from public or private research centers.
L'archive ouverte pluridisciplinaire HAL, est destinée au dépôt et à la diffusion de documents scientifiques de niveau recherche, publiés ou non, émanant des établissements d'enseignement et de recherche français ou étrangers, des laboratoires publics ou privés. 


\title{
Non-destructive evaluation of ceramic porosity using terahertz time-domain spectroscopy
}

\author{
Davit Hakobyan $^{\mathrm{a}}$, Maher Hamdi ${ }^{\mathrm{a}}$, Olivier Redon ${ }^{\mathrm{a}}$, Anthony Ballestero ${ }^{\mathrm{b}}$, Alexis Mayaudon ${ }^{\mathrm{b}}$, Laurence \\ Boyer $^{\mathrm{b}}$, Olivier Durand ${ }^{\mathrm{b}}$, Emmanuel Abraham ${ }^{\mathrm{c}}$ \\ ${ }^{a}$ CEA Tech Nouvelle Aquitaine, Cour Bersol Bâtiment B, 28 Avenue Gustave Eiffel, 33600 Pessac, France \\ ${ }^{b}$ Center for Technology Transfers in Ceramics, Parc Ester Technopole, 7 rue Soyouz, 87068 Limoges, France \\ ${ }^{c}$ Univ. Bordeaux, CNRS, LOMA, UMR 5798, F-33400 Talence, France
}

\begin{abstract}
Dielectric constants of $\mathrm{Al}_{2} \mathrm{O}_{3}$ (alumina) ceramic samples have been measured with non-destructive terahertz time-domain spectroscopy. Using an effective medium approximation, we demonstrated that the relative permittivity can be used to evaluate the porous alumina samples, with porosity fractions ranging from $0 \%$ to $20 \%$ per volume. This result makes it possible to control sample porosity, a key material parameter related to mechanical strength under compression load, hardness and wear resistance, or thermal stability. We also employed terahertz time-domain spectroscopy to differentiate alumina samples fabricated with different grain sizes, or reveal a small fraction of impurities, such as $5 \%$ vol of $\mathrm{ZrO}_{2}$, embedded in non-porous alumina. These results demonstrated the potential of terahertz radiation for non-destructive characterization of low loss high dielectric constant ceramics.
\end{abstract}

Keywords: Porosity evaluation, aluminum oxide $\mathrm{Al}_{2} \mathrm{O}_{3}$, ceramics, non-destructive evaluation, dielectric properties, terahertz time-domain spectroscopy.

\section{Introduction}

Bridging the boundary between the infrared and microwaves, terahertz $(\mathrm{THz})$ radiation encompasses electromagnetic frequencies from $0.1 \mathrm{THz}$ to roughly $10 \mathrm{THz}$. Its unique properties, such as good penetration through various non-conductive materials, low scattering, free-space propagation, low photon energy, and broad spectral bandwidth, encouraged the development of $\mathrm{THz}$ photonics [1]. To this end, one needs $\mathrm{THz}$ effective functional systems with improved quasi-optical components including mirrors and lenses, attenuators, isolators, modulators, directional couplers, etc. Ceramic dielectric materials, with high permittivity and low losses, could be selected for promising applications in the $\mathrm{THz}$ spectral region.

Main previous works concerning the study of ceramic materials with $\mathrm{THz}$ radiation were oriented towards the characterization of their dielectric properties within the relatively transparent

Email addresses: maher.hamdi@cea.fr (Maher Hamdi), emmanuel.abraham@u-bordeaux.fr (Emmanuel Abraham)
$0-3 \mathrm{THz}$ spectral window. In 2003, Bolivar et al. measured the dielectric constant and loss tangent of steatite, aluminium oxide (alumina), titania loaded polystyrene and zirconium-tin-titanate [2]. Among many other studies, one can mention broadband dielectric characterization of alumina $[3,4,5,6,7,8,9]$, boron nitride [10], low temperature co-fired ceramics based on willemite [11], and fiber-reinforced oxide-oxide ceramic matrix composites [12].

The porosity of a material is defined as the fraction of the volume of voids - or empty spaces - over the total volume. A key parameter in ceramic material production concerns the control of this porosity, since it is related to several physical properties, such as mechanical strength under compression load, hardness and wear resistance, or thermal stability. The evaluation of ceramic porosity can be achieved by boiling method or mercury injection $[13,14]$. Both techniques require sample destruction and sample contact. Several non-destructive approaches are reported in the literature using ultrasounds, X-rays, infrared waves or a split cylin- 
der [15]. Both split cylinder and ultrasonic techniques require sample contact. X-rays are harmful and additional resources are required to ensure the operator safety. Infrared-based techniques are sensitive to environment and, to obtain quantitative results, additional theoretical analysis are required. In contrast, $\mathrm{THz}$ radiation can propose an effective contact-less and non-destructive approach for the characterization and control of ceramic porosity. Recently, using zirconium dioxide ceramic, Ye and co-workers proposed an in-situ evaluation of porosity in thermal barrier coatings based on temporal broadening of $\mathrm{THz}$ pulses [16]. They demonstrated that, as the porosity increased, the refractive index decreased and the extinction coefficient increased. They also emphasized that the relative temporal broadening ratio could be obtained faster and in an easier manner compared to the dielectric parameters in both the transmission and reflection modes.

In this paper, we focused our attention on alumina since it is the most important, widely used and cost effective oxide ceramic material. After presenting the sample manufacturing procedure and characterization, we investigated the $\mathrm{THz}$ dielectric properties of alumina ceramic samples with porosity fractions equal to $0 \%_{\mathrm{vol}}, 5 \%_{\mathrm{vol}}, 10 \%_{\mathrm{vol}}, 15 \%_{\mathrm{vol}}$ and $20 \%$ vol. Using an effective medium approximation, we found a simple relationship between the real part of the relative permittivity and the sample porosity. We also demonstrated that $\mathrm{THz}$ timedomain spectroscopy can be effective to reveal a small fraction of impurities, such as $5 \%$ vol of $\mathrm{ZrO}_{2}$ powder embedded in non-porous alumina.

\section{Experimental procedures}

\subsection{Sample fabrication and characterization}

Pure alumina samples were prepared by CTTC (Center for Technology Transfers in Ceramics) with two different $\mathrm{Al}_{2} \mathrm{O}_{3}$ powders with grain size distribution $d_{50}=1 \mu \mathrm{m}$ and $d_{50}=9.3 \mu \mathrm{m}$ (median diameters). The samples exhibit a porosity ranging from 0 (bulk material) to $20 \%$ vol with an increment of $5 \% \mathrm{vol}$. The powder mixtures were composed of $\mathrm{Al}_{2} \mathrm{O}_{3}$ granulated powder and a proper volume of spherical organic pore formers to control the final porosity of the material. The pore former sphere diameter was controlled by scanning electron miroscopy (SEM) with a JEOL 6360 LA microscope with a $\times 5$ to $\times 60000$ magnification and

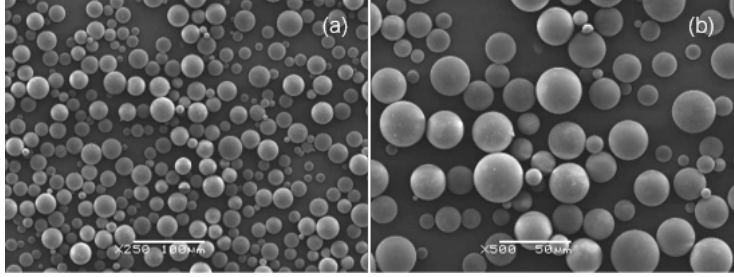

Figure 1: SEM pictures of spherical organic pore formers used to prepare $\mathrm{Al}_{2} \mathrm{O}_{3}$ porous samples. (a) Low magnification (white bar: $100 \mu \mathrm{m}$ ). (b) High magnification (white bar: $50 \mu \mathrm{m})$.

$3 \mathrm{~nm}$ resolution. The sphere diameters range from 1 to $50 \mu \mathrm{m}$, as shown in Fig. 1. After debinding and sintering, the remaining pore geometry is therefore expected to be spherical.

To simulate a material phase inclusion or pollution in the sample, CTTC prepared an additional $\mathrm{Al}_{2} \mathrm{O}_{3}$ sample with $\mathrm{ZrO}_{2}$ impurities. This sample is non-porous and thus contains $0 \%$ vol theoretical volume porosity. The powder mixtures were composed of $\mathrm{Al}_{2} \mathrm{O}_{3}$ granulated powder $\left(d_{50}=1 \mathrm{\mu m}\right.$ grain size) and $5 \%_{\text {vol }}$ of $\mathrm{ZrO}_{2}$ powder. No pore former spheres were added in this case.

Due to isostatic pressing requirements, the ceramic powders $\left(\mathrm{Al}_{2} \mathrm{O}_{3}\right.$ and $\left.\mathrm{Al}_{2} \mathrm{O}_{3} / \mathrm{ZrO}_{2}\right)$ needed to be granulated to improve the mould filling and the microstructural homogeneity of the final samples. To do so, a freeze granulation process was performed, consisting in a pulverization of ceramic particle suspension droplets into liquid nitrogen followed by the sublimation of frozen solvent. All the powder mixtures were shaped by isostatic pressing with a $232 \mathrm{kN}$ uniaxial press and a $30 \mathrm{~mm}$ in diameter stainless steel mould. The parts are then debinded and sintered under air from $1500^{\circ} \mathrm{C}$ to $1700^{\circ} \mathrm{C}$ in a muffle furnace. Final samples consist in pellets with $25 \mathrm{~mm}$ in diameter and $1 \mathrm{~mm}$ thickness after sintering. The porosity of each sample was characterized by the mercury porosimetry technique with a Micromeritics Autopore IV9510 allowing the detection of 0.003 to $360 \mu \mathrm{m}$ pore size diameter. The microstructure and surface topography of the samples were controlled by SEM observations.

\subsubsection{From 0 to $20 \%$ vol $\mathrm{Al}_{2} \mathrm{O}_{3}$ porous samples with $d_{50}=1 \mu \mathrm{m}$ powder}

The mercury porosimetry analysis were performed on the $d_{50}=1 \mathrm{\mu m} \mathrm{Al}_{2} \mathrm{O}_{3}$ samples with porosities equals to $0,5,10,15$ and $20 \%_{\text {vol }}$ (Fig. 2). 

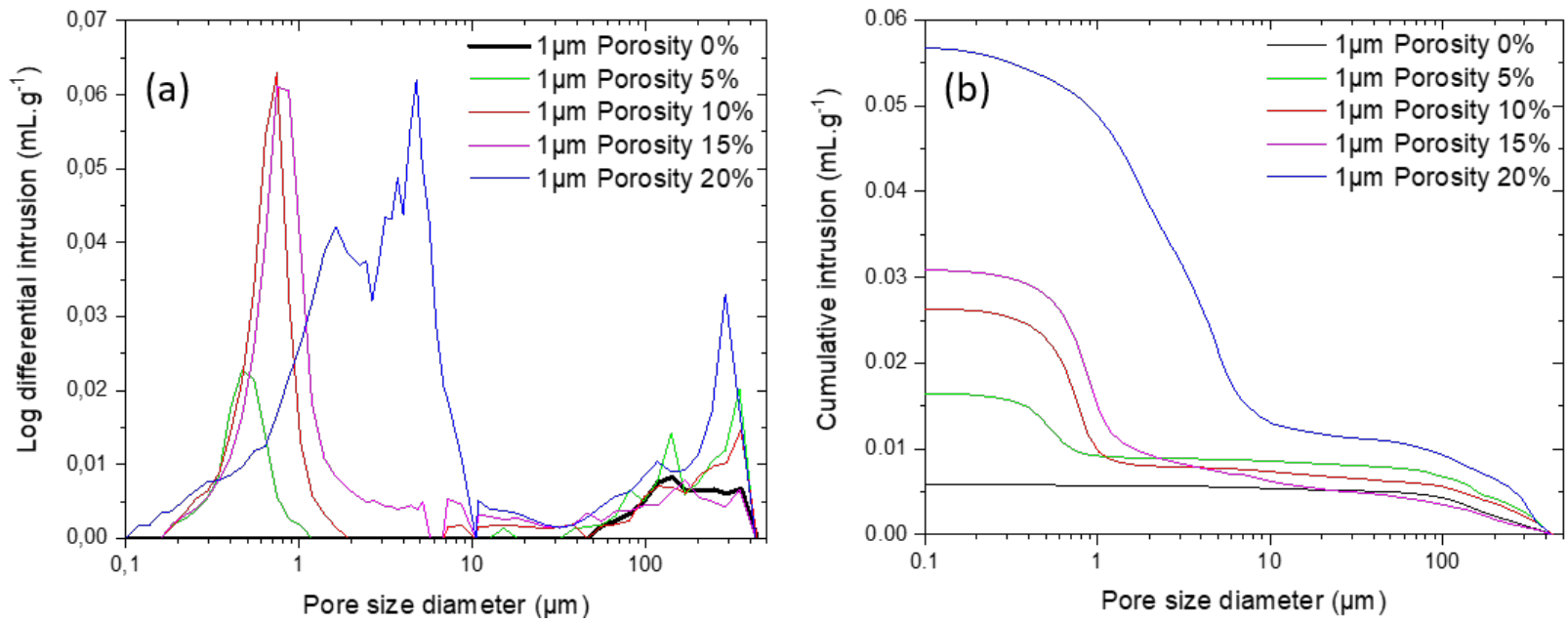

Figure 2: Mercury porosimetry analysis. Log of differential intrusion (a) and cumulative intrusion (b) as a function of pore size diameter for $d_{50}=1 \mathrm{\mu m} \mathrm{Al}_{2} \mathrm{O}_{3}$ samples with 0 to $20 \%$ vol theoretical porosity.
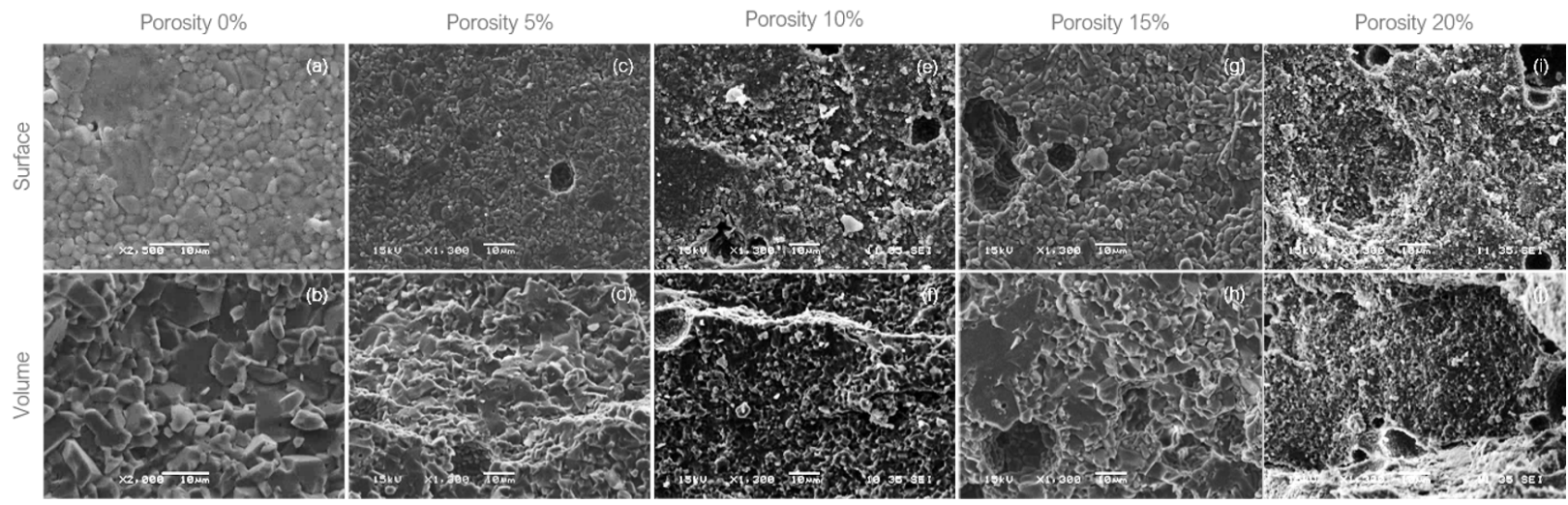

Figure 3: High magnification SEM pictures (surface and volume) for $d_{50}=1 \mu \mathrm{m}$ of $\mathrm{Al}_{2} \mathrm{O}_{3}$ samples with $0 \%_{v o l}(\mathrm{a}, \mathrm{b}), 5 \%$ vol $(\mathrm{c}, \mathrm{d})$, $10 \%_{\text {vol }}(\mathrm{e}, \mathrm{f}), 15 \%_{\text {vol }}(\mathrm{g}, \mathrm{h})$ and $20 \%_{\text {vol }}(\mathrm{i}, \mathrm{j})$ porosity levels. White bar: $10 \mu \mathrm{m}$.

In this figure, and for all mercury porosimetry analysis performed in this paper, the minimum pore size diameter has been fixed to $0.1 \mu \mathrm{m}$ since no porosity has been detected below this pore size. First, we notice that the $0 \%$ vol sample does not present any pore size diameter domain below $50 \mu \mathrm{m}$, visible on the log differential intrusion graphs (Fig. 2(a)). However, it appears a pore size diameter domain above $100 \mu \mathrm{m}$, indicating a remaining portion of voids in the sample. We can thus conclude that the $0 \%$ vol sample does not exactly correspond to bulk alumina material. Then, the profiles of the 5,10 and $15 \%$ vol porosity curves look similar with three different pore size diameter domains from 0.1 to $2 \mu \mathrm{m}, 2$ to $100 \mu \mathrm{m}$ and above $100 \mu \mathrm{m}$. We believe that the $0.1-2 \mu \mathrm{m}$ pore size diameter domain could be correlated to an inter/intra granular porosity or pore former spheres broken in smaller pieces during the shaping process. Nevertheless, the contribution of an inter/intra granular porosity could be more significant with the pore former content, as shown in Fig. 2(b). Indeed, to keep the spherical pore shape and to avoid material collapsing, the thermal treatment temperature has to be decreased with the increasing of pore former quantity. This modification could create lack of sintering in the ceramic pellets. The $2-100 \mu \mathrm{m}$ domain is directly related to the size of pore former spheres introduced in the powder mixture. For the $15 \%$ vol and $20 \%$ vol $\mathrm{Al}_{2} \mathrm{O}_{3}$ porous samples, the contribution of $0.1-2 \mu \mathrm{m}$ pore size diameter domain decreases to the benefit of the two other domains $(2-10 \mu \mathrm{m}$ and above $100 \mu \mathrm{m})$, 

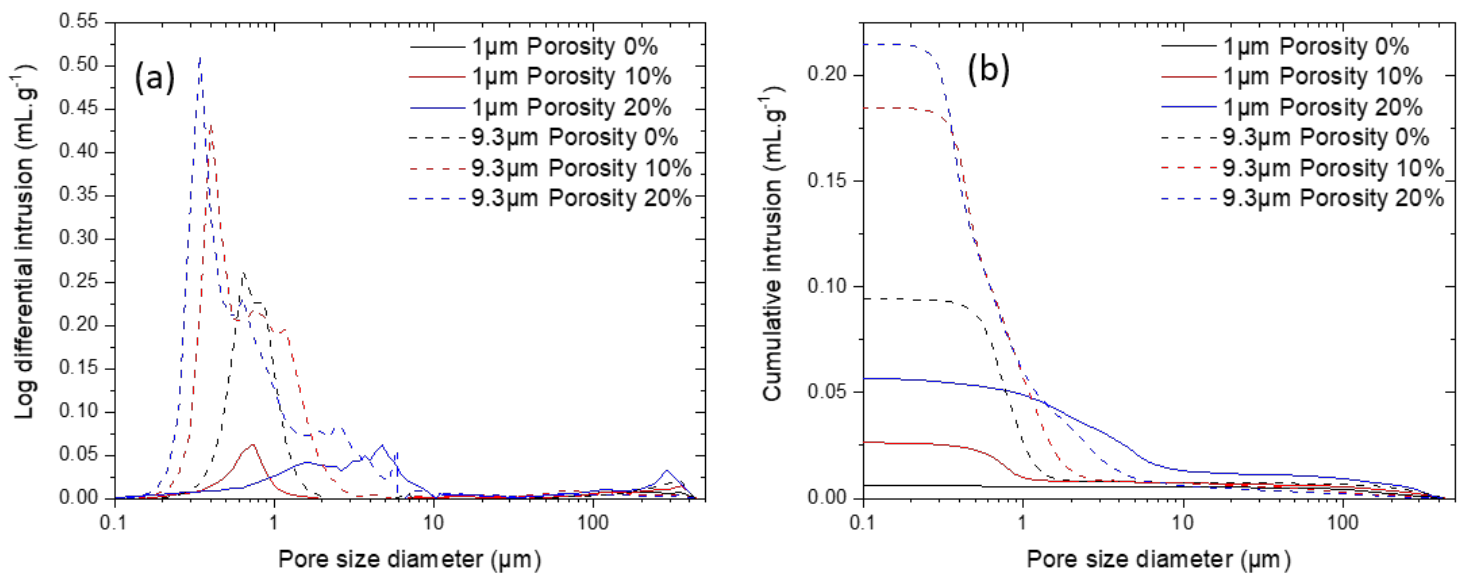

Figure 4: Mercury porosimetry analysis. Log of differential intrusion (a) and cumulative intrusion (b) as a function of pore size diameter. Comparison between $\mathrm{Al}_{2} \mathrm{O}_{3}$ samples with 0,10 and $20 \%$ vol theoretical porosity, fabricated from $d_{50}=1 \mu \mathrm{m}$ and $d_{50}=9.3 \mu \mathrm{m}$ powders.

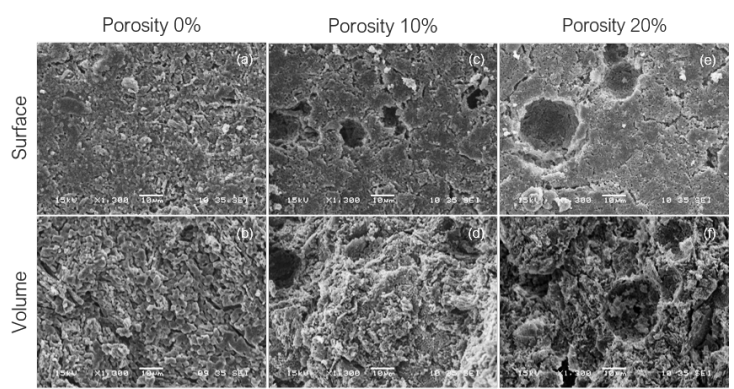

Figure 5: High magnification SEM pictures (surface and volume) for $d_{50}=9.3 \mu \mathrm{m}$ of $\mathrm{Al}_{2} \mathrm{O}_{3}$ samples with $0 \%$ vol $(\mathrm{a}, \mathrm{b})$, $10 \%_{v o l}(\mathrm{c}, \mathrm{d}), 20 \%_{\text {vol }}(\mathrm{e}, \mathrm{f})$ porosity levels. White bar: $10 \mu \mathrm{m}$.

as shown in Fig. 2(a) (purple and blue curves). This phenomenon is amplified for the $20 \%$ vol sample and maybe related to a decreasing rate of broken pore former spheres. Their integrity seems to be preserved by a lower amount of ceramic material contained in the $20 \% \mathrm{vol}$ of porosity powder mixture.

The porosity modification is highlighted by the high magnification SEM observation of the surface and volume of the five samples (Fig. 3). The voids and pore concentration clearly increase with the amount of pore former volume. The remaining porosity in the sintered part is modified, as shown by the SEM analysis (Fig. 3). The only pore size diameter domain shared by all the samples is the one above $100 \mu \mathrm{m}$, which is not supposed to be present. This should indicate cracks or fractures in all the samples which can appear during the shaping or the several thermal treatments. This hypothesis can be discarded by the SEM analysis carried on the samples (Fig. 3). Indeed, the high magnification SEM analysis, whatever the sample specification is, do not reveal the presence of any cracks on the surface or in the volume. To perform mercury porosimetry and SEM analysis, the pellets were broken and the mechanical stress may induce defect apparition above $100 \mu \mathrm{m}$.

It is important to note that SEM and mercury porosimetry represent destructive characterization techniques. The increase of the spherical pore concentration is the consequence of the introduction of larger spherical pore formers. Moreover, the very rugged profiles seen in the sample volumes (Fig. 3(f,j)) correspond to fracture faces which may cause porosity in the samples above $100 \mathrm{\mu m}$. Comparatively, we have to point out that the following analysis with $\mathrm{THz}$ radiation presented in this paper, will not undergo any sample stress or destruction.

\subsubsection{From 0 to $20 \%$ vol porosity $\mathrm{Al}_{2} \mathrm{O}_{3}$ samples with $d_{50}=9.3 \mu \mathrm{m}$ powder}

For each porosity, the $d_{50}=9.3 \mu \mathrm{m}$ sample pellets were shaped, debinded and sintered in the same conditions than the $d_{50}=1 \mu \mathrm{m}$ ones. The mercury porosimetry analysis were performed on the 0,10 an $20 \%$ vol porosity $\mathrm{Al}_{2} \mathrm{O}_{3}$ samples and compared with those of the $d_{50}=1 \mu \mathrm{m}$ samples, as shown in Fig. 4. For the samples made with the 

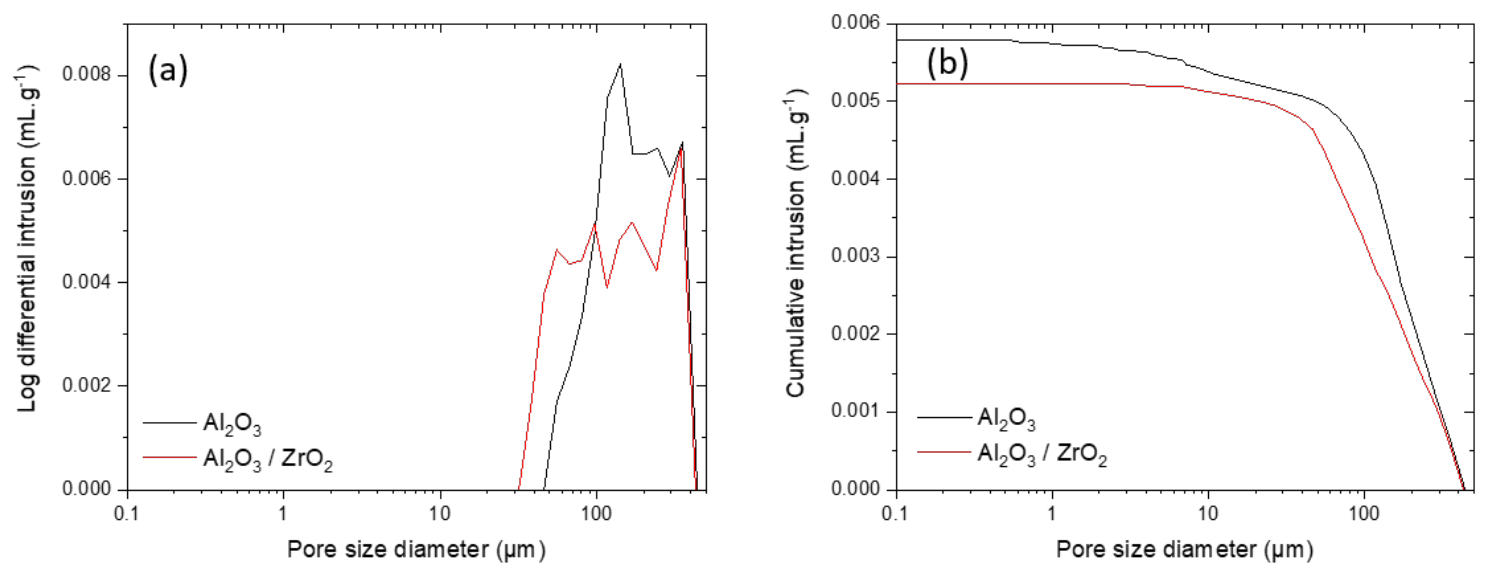

Figure 6: Mercury porosimetry analysis. Log of differential intrusion (a) and cumulative intrusion (b) as a function of pore size diameter for $0 \%$ vol porosity $d_{50}=1 \mu \mathrm{m} \mathrm{Al}_{2} \mathrm{O}_{3}$ and $\mathrm{Al}_{2} \mathrm{O}_{3}-\mathrm{ZrO}_{2}$ mixture.

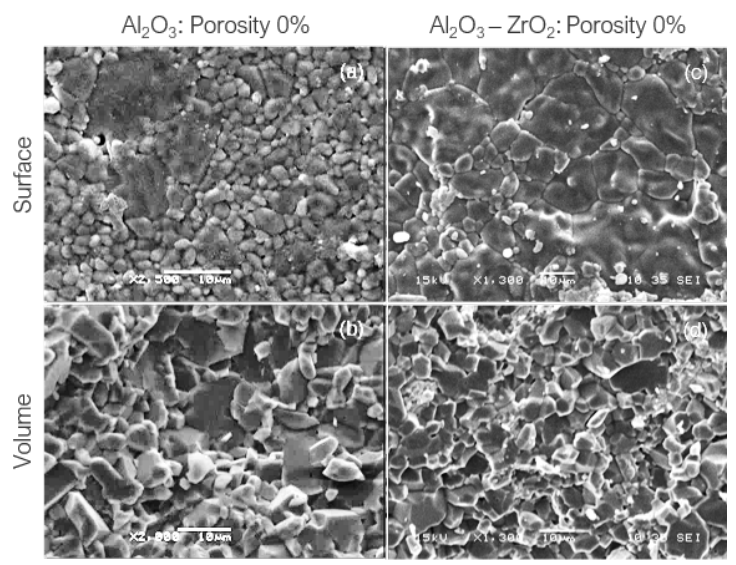

Figure 7: High magnification SEM pictures (surface and volume) for $d_{50}=1 \mu \mathrm{m}$ of $\mathrm{Al}_{2} \mathrm{O}_{3}$ samples with $0 \%_{\mathrm{vol}}(\mathrm{a}, \mathrm{b})$ and $\mathrm{Al}_{2} \mathrm{O} 3-\mathrm{ZrO}_{2}(\mathrm{c}, \mathrm{d})$ mixture samples. White bar: $10 \mu \mathrm{m}$.

$9.3 \mu \mathrm{m}$ powder, the introduced mercury volume is clearly higher for all porosities. The samples show a $30 \%$ porous volume increase compared to the $1 \mu \mathrm{m}$ powder. The very low porosity at high pore size diameter above $100 \mu \mathrm{m}$ is again due to the preparation procedure for sample characterization). A 0.3 to $2 \mu \mathrm{m}$ pore size diameter domain, which is not supposed to be present, appears on the profile of the $d_{50}=9.3 \mu \mathrm{m} 0 \% \mathrm{vol}$ porosity (Fig. $4(\mathrm{a})$, black dash curve) and is absent on the $d_{50}=1 \mu \mathrm{m} 0 \%$ vol porosity profile (Fig. 4(a), black curve). This phenomenon is not due to an addition of pore formers since no spheres were introduced in this case, but related to an inter/intra granular porosity caused by a lack of sintering. The $d_{50}=9.3 \mu \mathrm{m} 10 \%$ vol and $20 \%$ vol porosity samples (Fig. 4(a), red dash and blue dash curves, respectively) show a shift towards smaller and bigger pore size diameters, respectively due to inter/intragranular porosity and pore formers spheres introduction. The pore size diameters and porous volume evolutions of these two samples are similar those of $d_{50}=1 \mu \mathrm{m} 10 \% \mathrm{vol}$ and $20 \%$ vol porosity samples (Fig. 4(a), red and blue curves, respetively) down to the final porosity (Fig. 4(b)).

We also used high magnification SEM images to analyze the porosity modification of the surface and volume of the three samples (Fig. 5). Again, the voids and pore concentration increase with the amount of introduced pore formers. Indeed, the spherical pore concentration increase is the sign of a larger pore former sphere introduction. The lack of sintering and consolidation is still present and observed whatever the pore former sphere amount introduced. The comparison of SEM images in Figs. 3(a,b) and 5(a,b) exhibits the remaining porosity and the different microstructures between $d_{50}=9.3 \mathrm{\mu m} 0 \%$ vol porosity and $d_{50}=1 \mu \mathrm{m} 0 \%_{\text {vol }}$ porosity. Indeed, full sintering of larger $\mathrm{Al}_{2} \mathrm{O}_{3}$ grain size can be harder to achieve inducing a high residual porosity and a lack of consolidation. 


\subsection{3. $0 \%$ vol $\mathrm{Al}_{2} \mathrm{O}_{3}$ and $\mathrm{Al}_{2} \mathrm{O}_{3}-5 \%$ vol $\mathrm{ZrO}_{2}$ mixture samples with $d_{50}=1 \mu \mathrm{m}$ powder}

The mercury porosimetry analysis of $\mathrm{Al}_{2} \mathrm{O}_{3}$ and $\mathrm{Al}_{2} \mathrm{O}_{3}-\mathrm{ZrO}_{2}$ mixture samples are very similar (Fig. 6). The very low porosity at high pore size diameter is again due to the preparation procedure for sample characterization. The presence of a secondary phase cannot be detected by mercury porosimetry but some inclusions are revealed by SEM images, as shown in Fig. 7. Indeed, the whiter and clearer grains or areas correspond to dispersed $\mathrm{ZrO}_{2}$ material in the $\mathrm{Al}_{2} \mathrm{O}_{3}$ matrix. No cracks are detected on the surface or in the volume of the pellets.

\subsection{THz time-domain spectroscopy}

To measure the dielectric properties of the samples, we used THz time-domain spectroscopy. This technique can provide both amplitude and phase information of $\mathrm{THz}$ radiation transmitted by a sample in a single measurement and does not require the use of Kramers-Krönig relations to extract both real and imaginary parts of the dielectric permittivity [17]. Moreover, THz time-domain spectrometers are now commercially available with high detection sensitivity and large spectral range, contributing to the development of a valuable material $\mathrm{THz}$ database.

For our study, we used a commercially available $\mathrm{THz}$ time-domain spectrometer (TeraFlash, Toptica Photonics). The THz beam is sent onto a sample with a beam diameter of $2.5 \mathrm{~mm}$ to ensure a large and homogenized illumination. The system measures a signal proportional to the temporal evolution of the $\mathrm{THz}$ electric field transmitted by the sample with a dynamic range of $100 \mathrm{~dB}$ and a spectral bandwidth up to $6 \mathrm{THz}$. Owing to a highly precise mechanical delay stage, the system acquires up to 60 temporal waveforms per second along a $50 \mathrm{ps}$ scan range. The entire setup was enclosed inside a dry air injected chamber to eliminate the absorption of $\mathrm{THz}$ radiation by ambient water vapor.

Using fast Fourier transform, temporal data are transformed into the frequency domain. The ratio between sample and reference signals provides the complex function, from which one can extract the complex relative permittivity $\tilde{\varepsilon}_{r}=\varepsilon_{r}-i \varepsilon_{r}^{\prime}$ as a function of the THz frequency [17]. From this procedure, one can obtain the $\mathrm{THz}$ dielectric properties of the sample, represented for instance by the relative permittivity $\varepsilon_{r}$ and the dielectric loss tangent defined as:

$$
\tan \delta=\frac{\varepsilon_{r}}{\varepsilon_{r}^{\prime}} .
$$

The measurement procedure is as follows. For a given sample family (same porosity fraction, same grain size, same composition), three different alumina samples have been studied to reduce uncertainty coming from both sample fabrication and experimental measurement. For each sample, we precisely measured the pellet thickness, by means of a micrometer caliper with an accuracy of up to $5 \mu \mathrm{m}$ and with a ratchet stop, before extracting the average relative permittivity and loss tangent. Simultaneously, we also recorded a reference signal obtained without sample. The three consecutive sample measurements make it possible to calculate the average and standard deviation of both relative permittivity and loss tangent. It should be also mentioned that mercury porosimetry only captures open pores whereas $\mathrm{THz}$ spectroscopy is more accurate providing a measurement that reflects both open and closed pores.

\section{Results and discussion}

\section{1. $\mathrm{THz}$ dielectric properties of $\mathrm{Al}_{2} \mathrm{O}_{3}$ porous sam- ples with $d_{50}=1 \mu \mathrm{m}$ powder}

In this first experiment, we studied the dielectric properties of $\mathrm{Al}_{2} \mathrm{O}_{3}$ samples composed of $d_{50}=$ $1 \mathrm{\mu m}$ grain size with porosity fractions equal to 5 , 10,15 and $20 \%$ vol.

For these porosity fractions, Fig. 8 shows the evolution of both the relative permittivity (a) and the loss tangent (b) as a function of frequency from 0.3 to $2 \mathrm{THz}$. Above this latter frequency, the signal-to-noise ratio is too small owing to important absorption of $\mathrm{THz}$ radiation by the samples. In the figure, the markers indicate the average values over three samples fabricated under same conditions. The color shade backgrounds are the standard deviations over the three samples.

First, as shown in Fig. 8(a), the relative permittivities of the different samples are all distributed between 7.5 and 9.3 , in agreement with previous studies $[2,3,4,5,7,8]$, even if it is difficult to compare separate studies since the fabrication protocol and porosity fraction can strongly differ. Then, for all porosity fractions, we observe that the relative permittivity increases as a function of $\mathrm{THz}$ frequency. This is especially remarkable considering 

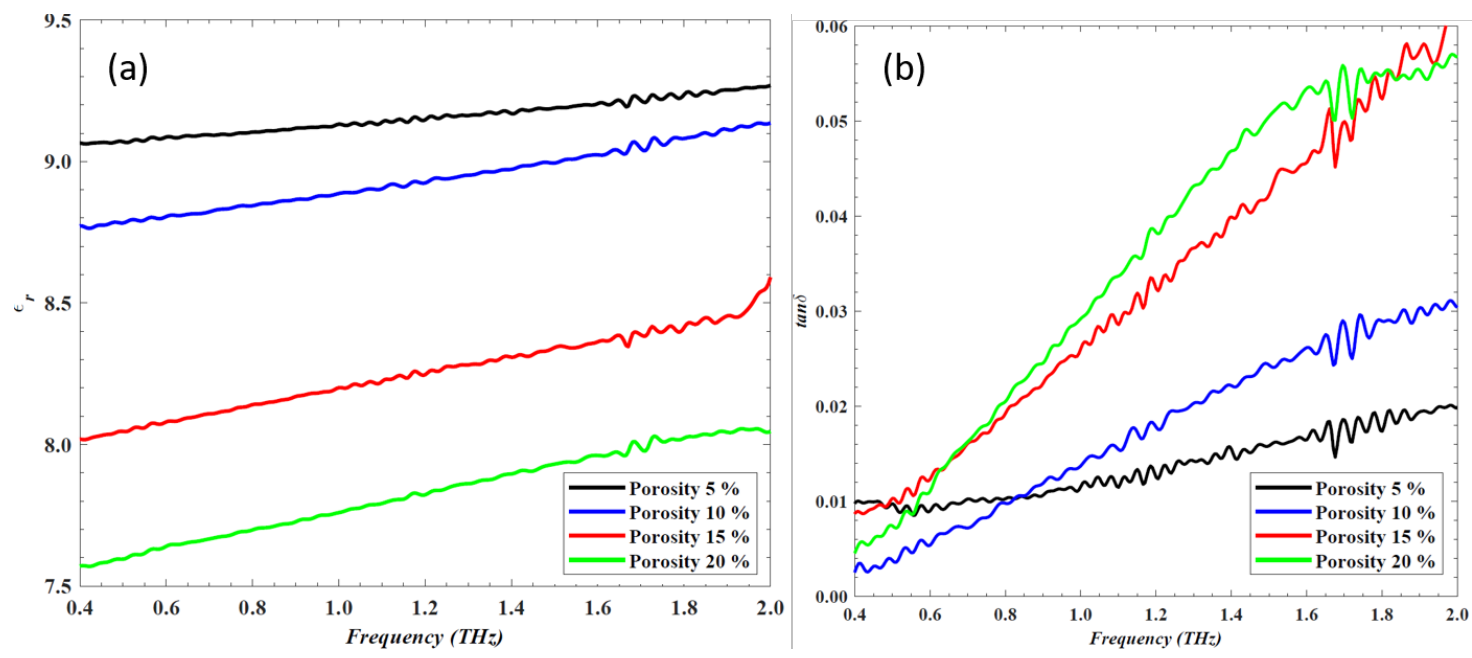

Figure 8: Dielectric properties of $d_{50}=1 \mathrm{\mu m}$ grain size alumina samples with porosity fractions equal to $5 \%, 10 \%, 15 \%$ and $20 \%$. (a) Relative permittivity. (b) Loss tangent. Markers correspond to the average values measured over three different samples.

samples with higher porosity. We observe a similar behavior for the dielectric loss tangent (Fig. 8(b)). It is around 0.005 below $0.5 \mathrm{THz}$, confirming that alumina ceramic can be considered as a low loss high dielectric constant material, in agreement with previous studies $[2,3,4,5,7,8]$. The loss tangent also gradually increases as a function of $\mathrm{THz}$ frequency. Similarly, this trend is more remarkable considering samples with higher porosity. For example, the dielectric loss tangent of alumina sample with $20 \%$ porosity is around 0.003 at $0.3 \mathrm{THz}$ but nearly 20 times larger at $2 \mathrm{THz}$. Since higher frequencies are related to shorter wavelengths $(1 \mathrm{THz}$ corresponds to a wavelength of $0.3 \mathrm{~mm}$ ), this trend may be attributed to higher scattering of $\mathrm{THz}$ radiation by the samples at high frequencies. $\mathrm{THz}$ scattering can also explain the increase of loss tangent as a function of sample porosity due to the presence of larger voids or empty spaces between alumina grains.

Regarding differences of dielectric properties between samples with different porosity fractions, we observe a decrease of the relative permittivity for samples with higher porosity, as shown in Fig. 8(a). Globally, we observe a $15 \%$ decrease of the sample permittivity as the porosity fraction changes from $5 \%$ to $20 \%$. We note a qualitatively similar behavior for the dielectric loss tangent above $0.5 \mathrm{THz}$. It strongly increases for the samples with higher porosity fractions (Fig. 8(b)). However, this trend is not properly preserved for lower frequencies than

\section{$0.5 \mathrm{THz}$.}

\subsection{Effective medium approximation description}

The effective medium theory (EMT) has been used for over a century to study the optical, electric, magnetic and mechanical properties of materials $[18,19]$. With $\mathrm{THz}$ radiation, EMT has been used to characterize pellets composed of polyethylene powder and specific molecules such as explosives, drugs, etc. We can also cite the $\mathrm{THz}$ detection of porosity in pharmaceutical tablets [20]. The mathematical equations describing different transport phenomena (dielectric permittivity, electrical conductivity, etc.) in each of these fields are interchangeable. At the macroscopic scale, the effective properties of a multi-phase composite material can be accurately computed using the properties and the relative fractions of its components. The complex effective permittivity of a heterogeneous medium in the $\mathrm{THz}$ range can be estimated from effective medium models (EMMs). The most well-known one is the Maxwell-Garnett model, which is limited to spherical inclusion embedded in a host material with a low volume fraction [21]. Many studies have been performed to extend the effective model to higher volume fractions and handling more complicated shapes of inclusions [22, 23, 24, 25].

In this section, we evaluate the applicability of different EMMs on our experimental data. Each model gives the relationship between the complex 

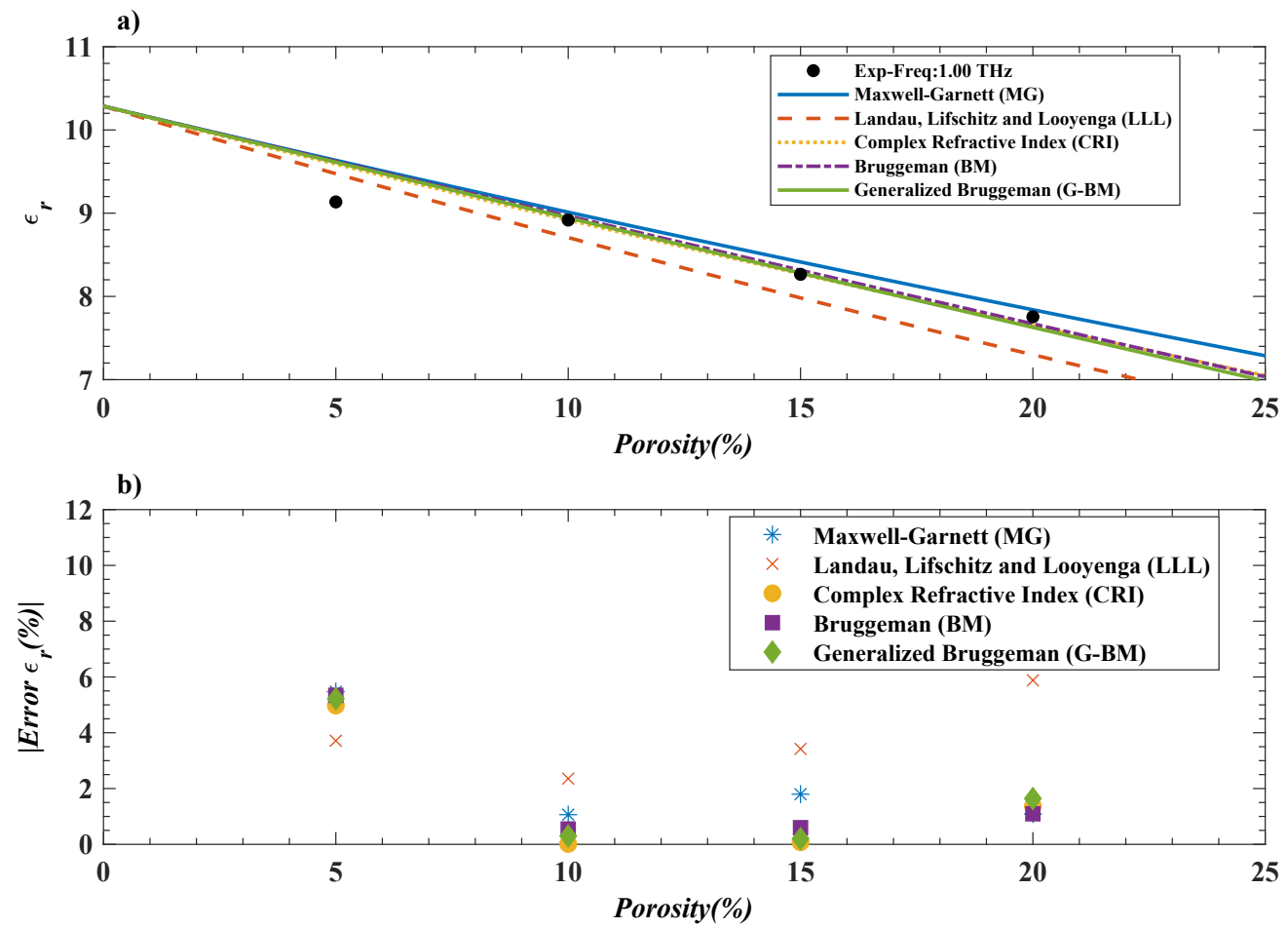

Figure 9: (a) Comparison between experimental relative permittivity of the samples (black dots) and the one predicted from EMMs (solid lines), at $1 \mathrm{THz}$ and as a function of the sample porosity fraction. To estimate the $0 \%_{\text {vol }}$ relative permittivity we applied the ZPA calculation. (b) Absolute error between experimental data and the EMM simulations as a function of the sample porosity fraction.

relative permittivity of the effective medium $\tilde{\varepsilon}_{r}$ and those of the $\mathrm{Al}_{2} \mathrm{O}_{3}$ host material and the embedded spherical inclusions (air or pores). These equations are as follows.

- Maxwell-Garnett model (MG) [21]:

$$
\left(\frac{\tilde{\varepsilon}_{r}-\tilde{\varepsilon}_{0}}{\tilde{\varepsilon}_{r}+2 \tilde{\varepsilon}_{0}}\right)=\phi_{1}\left(\frac{\tilde{\varepsilon}_{1}-\tilde{\varepsilon}_{0}}{\tilde{\varepsilon}_{1}+2 \tilde{\varepsilon}_{0}}\right),
$$

where $\tilde{\varepsilon}_{0}$ and $\tilde{\varepsilon}_{1}$ are the complex relative permittivities, respectively, of the $\mathrm{Al}_{2} \mathrm{O}_{3}$ host material and pore guest material, and $\phi_{1}$ is the volume fraction of the pore guest. In this model, the host and guest materials are not exchangeable. As a consequence, the value of $\tilde{\varepsilon}_{r}$ is very sensitive to the difference between $\tilde{\varepsilon}_{0}$ and $\tilde{\varepsilon}_{1}$, and also to a high volume fraction of pore inclusions [26].

- Bruggeman model (BM) [27]:

$$
\phi_{1}\left(\frac{\tilde{\varepsilon}_{1}-\tilde{\varepsilon}_{r}}{\tilde{\varepsilon}_{1}+2 \tilde{\varepsilon}_{r}}\right)+\phi_{2}\left(\frac{\tilde{\varepsilon}_{2}-\tilde{\varepsilon}_{r}}{\tilde{\varepsilon}_{2}+2 \tilde{\varepsilon}_{r}}\right)=0,
$$

where $\tilde{\varepsilon}_{1}$ and $\tilde{\varepsilon}_{2}$ are the complex relative permittivities of the material 1 ( $\mathrm{Al}_{2} \mathrm{O}_{3}$ host) and material
2 (pore guest), $\phi_{1}$ and $\phi_{2}$ their volume fractions, respectively, with the relation $\phi_{1}+\phi_{2}=1$.

For ellipsoidal voids, the equation is modified as follows (generalized BM model, noted G-BM):

$\phi_{1}\left(\frac{\tilde{\varepsilon}_{1}-\tilde{\varepsilon}_{r}}{\tilde{\varepsilon}_{1}+L\left(\tilde{\varepsilon}_{1}-\tilde{\varepsilon}_{r}\right)}\right)+\phi_{2}\left(\frac{\tilde{\varepsilon}_{2}-\tilde{\varepsilon}_{r}}{\tilde{\varepsilon}_{2}+L\left(\tilde{\varepsilon}_{2}-\tilde{\varepsilon}_{r}\right)}\right)=0$,

where $\mathrm{L}$ is a depolarization factor, with $L=1 / 3$ for spherical voids $[27,28,29]$. This model can be applied with contrasted values of $\tilde{\varepsilon}_{1}$ and $\tilde{\varepsilon}_{2}$ and any volume fractions [26].

- Landau, Lifschitz and Looyenga model (LLL) [22]:

$$
\sqrt[3]{\tilde{\varepsilon}_{r}}=\phi_{1} \sqrt[3]{\tilde{\varepsilon}_{1}}+\phi_{2} \sqrt[3]{\tilde{\varepsilon}_{2}}
$$

where $\tilde{\varepsilon}_{1}$ and $\tilde{\varepsilon}_{2}$ are again the complex relative permittivities of the material $1\left(\mathrm{Al}_{2} \mathrm{O}_{3}\right.$ host $)$ and material 2 (pore guest), $\phi_{1}$ and $\phi_{2}$ their volume fractions, respectively, with the relation $\phi_{1}+\phi_{2}=1$. This model is well-suited for arbitrarily shaped particles. 

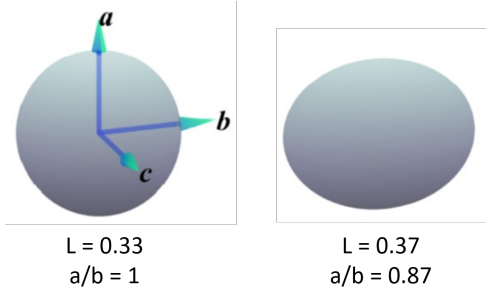

Figure 10: Dependency of the inclusion shape on the depolarization factor $\mathrm{L}$ and the aspect ratio $a / b$. Left: spherical intrusion. Right: ellipsoidal inclusion with $\mathrm{L}$ and $a / b$ calculated from Eqs. 4 and 7, respectively.

- Complex Refractive Index model (CRI) [22, $30]$.

$$
\sqrt{\tilde{\varepsilon}_{r}}=\phi_{1} \sqrt{\tilde{\varepsilon}_{1}}+\phi_{2} \sqrt{\tilde{\varepsilon}_{2}},
$$

where $\tilde{\varepsilon}_{1}$ and $\tilde{\varepsilon}_{2}$ are the complex relative permittivities of the material 1 ( $\mathrm{Al}_{2} \mathrm{O}_{3}$ host) and material 2 (pore guest), $\phi_{1}$ and $\phi_{2}$ their volume fractions, respectively, with the relation $\phi_{1}+\phi_{2}=1$. This empirical model is well-suited for porous material.

With these five EMMs, we can calculate the theoretical relative permittivities of the effective medium $\varepsilon_{r}$ as a function of the porosity fraction and compare the results to the experimental data already shown in Fig. 8(a). Moreover, EMMs assume that scattering of $\mathrm{THz}$ radiation is negligible [20]. Since we did not observe any significant broadening of the $\mathrm{THz}$ temporal waveforms for all the samples, we can conclude that scattering effects are not dominant in our work. This is also consistent with the $d_{50}=1 \mu \mathrm{m}$ grain size of pure alumina which is negligible compared to the wavelength of $\mathrm{THz}$ radiation. For different sample porosity fractions, Fig. 9(a) shows the experimental relative permittivity of the samples (black points) at $1 \mathrm{THz}$, extracted from the data presented in Fig. 8(a), together with the values predicted from the previous EMMs (lines). First, all models are in good agreement with the experimental data, even if the LLL one underestimates the value of the relative permittivity for porosity levels higher than $5 \% \mathrm{vol}$. Then, it is important to note, in the range from 0 to $25 \%$ vol, the linear dependency between the relative permittivity and the porosity, well reproduced by the EMM simulations. According to the fitting parameters, the linear dependency can be written as $\varepsilon_{r}=-0.13 P+10.25$, where $\mathrm{P}$ is the sample porosity fraction (in $\%$ vol).

Figure 9(b) shows that the absolute calculated error between experimental data and the EMMs sim-

\begin{tabular}{|c|c|c|c|}
\hline Porosity [\%] & \multicolumn{3}{|c|}{$\left[\varepsilon_{0}\right]$} \\
\cline { 2 - 4 } & ZPA & BM & MG \\
\hline 10 & 10.28 & 10.23 & 10.17 \\
\hline 15 & 10.28 & 10.22 & 10.1 \\
\hline 20 & 10.44 & 10.41 & 10.17 \\
\hline Average & 10.33 & 10.29 & 10.15 \\
\hline
\end{tabular}

Table 1: Relative permittivity $\varepsilon_{0}$ of pure $\mathrm{Al}_{2} \mathrm{O}_{3}$ host material estimated by the ZPA, BM and MG models at porosity levels equal to $10 \%_{\mathrm{vol}}, 15 \%_{\mathrm{vol}}$ and $20 \%_{\mathrm{vol}}$ at $1 \mathrm{THz}$. Last line: mean value for each model.

ulations stands in the 0 to $6 \%$ range. Again, we can observe that the predicted permittivities, except for the LLL model, are in good agreement with the experimental values. Considering the spherical aspect of pore formers, as previously shown in Fig. 1, we logically expect spherical inclusions. The LLL model, which does not consider any inclusion shape, cannot perform the accuracy of the other models. We suspect the minor discrepancy to the experimental data of the MG model is due to a slight deformation of the predicted spherical shaped inclusions. Logically, we can expect ellipsoidal inclusions with aspect ratio $a / b$, linked to the depolarization factor L, of the principle axis (a) and the two common axes (b,c) of a spheroid (Fig. 10) [31]. Using an iterative least-square fitting to the G-BM model (see Eq. 4) and the THz effective permittivities, the depolarization factor $\mathrm{L}$ has been estimated to 0.37 . Then, the aspect ratio $a / b$ can be estimated with the equation [31]:

$$
L=\frac{1}{1+1.6(a / b)+0.4(a / b)^{2}},
$$

which provides the result $a / b=0.87$, illustrated in Fig. 10 to better show the ellipsoid shape of the inclusions.

For a $5 \%$ vol porosity fraction, the significant discrepancy of all EMMs to the experimentally data can be explained by the large volume of the pore size between $10 \mu \mathrm{m}$ and $100 \mu \mathrm{m}$ (Fig. 2(b), green curve), leading to more scattering effects which are not considered in the currently used EMMs. We shall point out the absence of any pore size diameter domains in the log differential intrusion profiles (Fig. 2(a), black curve related to $1 \mu \mathrm{m}$ grain size and $0 \%$ vol porosity), at least, regarding to the resolution limit of the used mercury porosimetry system. 
Obviously, the good agreement of the experimental effective permittivity of the sample to theoretical curves confirms our assumption about the origin of the low accuracy of the EMMs at $5 \% \mathrm{vol}$ of porosity.

It is important to recall that the permittivities of the different components of the samples (host $\mathrm{Al}_{2} \mathrm{O}_{3}$ and pore guest materials) used in the EMM equations corresponds to pure bulk form. This bulk $\mathrm{Al}_{2} \mathrm{O}_{3}$ permittivity, corresponding to the real part of $\tilde{\varepsilon}_{0}$ in our EMMs, was calculated by considering the effective permittivities of the samples with known porosity fractions $\left(10 \%_{\mathrm{vol}}, 15 \%\right.$ vol and $20 \%$ vol $)$, the MG and BM models and the additional zero porosity approximation (ZPA) [32]. At porosity levels equal to $10 \% \mathrm{vol}, 15 \% \mathrm{vol}$ and $20 \% \mathrm{vol}$, Table 1 shows the relative permittivity $\varepsilon_{0}$ of pure $\mathrm{Al}_{2} \mathrm{O}_{3}$ host material, at $1 \mathrm{THz}$, estimated by ZPA, $\mathrm{BM}$ and MG models. The mean value of the relative permittivity across all models is close to the value for bulk alumina reported elsewhere, between 9.45 and 11.72 [33].

\subsection{Impact of the alumina powder grain size on $\mathrm{THz}$ dielectric properties}

In this second experiment, for porosity fractions equal to $0 \%, 10 \%$ and $20 \%$, we compared the dielectric properties of $\mathrm{Al}_{2} \mathrm{O}_{3}$ samples composed of $d_{50}=1 \mu \mathrm{m}$ grain size with those of $\mathrm{Al}_{2} \mathrm{O}_{3}$ samples composed of $d_{50}=9.3 \mu \mathrm{m}$ grain size. For all samples, the experimental procedure is similar to that described in the previous section, with again an averaging of the data provided by three different samples for a given grain size and porosity. For the different porosity fractions, Fig. 11 shows the evolution of the relative permittivity (a) and the loss tangent (b) as a function of frequency from 0.3 to $2 \mathrm{THz}$. Markers indicate the average values over three samples fabricated under same conditions and color shade backgrounds are the standard deviations. Experimental points corresponding to $d_{50}=1 \mu \mathrm{m}$ grain size, already plotted in Fig. 11, have been plotted again for comparison with those corresponding to $d_{50}=9.3 \mu \mathrm{m}$ grain size. First, the permittivity of $9.3 \mu \mathrm{m}$ grain size samples increases slower than that of $1 \mu \mathrm{m}$ grain size samples over the observed $\mathrm{THz}$ spectral range. Then, the permittivity of $9.3 \mu \mathrm{m}$ grain size samples is about 30 to $40 \%$ lower than that of $1 \mu \mathrm{m}$ grain size samples. This can be explained by the lower density of $9.3 \mu \mathrm{m}$ grain size sample with respect to that of $1 \mu \mathrm{m}$ grain size one.
For the $d_{50}=9.3 \mu \mathrm{m}$ grain size samples, the loss tangents exhibit large standard deviations due to larger $\mathrm{THz}$ absorption. Again, the dielectric loss tangent rapidly increases as a function of $\mathrm{THz}$ frequency, even if we note a more complex behavior for $9.3 \mu \mathrm{m}$ grain size samples. For example, we observe an oscillation of the loss tangent for the $9.3 \mu \mathrm{m}$ grain size sample with $10 \%$ porosity fraction, which was not observed for the $1 \mu \mathrm{m}$ grain size sample. Since standard deviations for $9.3 \mu \mathrm{m}$ grain size samples are also very large, it is difficult to go further with this observation. Moreover, the loss tangent of $1 \mu \mathrm{m}$ grain size samples is smaller than that of $9.3 \mu \mathrm{m}$ grain size samples over the considered $\mathrm{THz}$ frequency range, except the presence of some overlapping for the $20 \%$ porous samples above $1.5 \mathrm{THz}$. We attribute this behavior to the remaining porosity and the different microstructures between the two samples with a more difficult full sintering of larger $\mathrm{Al}_{2} \mathrm{O}_{3}$ grain size inducing higher residual porosity.

\subsection{Impact of impurity inclusions on $\mathrm{THz}$ dielec- tric properties}

The last experiment is slightly different since it does not concern the influence of sample porosity on $\mathrm{THz}$ dielectric constants, but rather the potential of $\mathrm{THz}$ time-resolved spectroscopy to detect an impurity in a sample. As explained before, the sample is non-porous and composed of $d_{50}=1 \mu \mathrm{m}$ grain size with $5 \%$ vol $\mathrm{ZrO}_{2}$ impurities.

$\mathrm{THz}$ dielectric constants were extracted with the similar approach as before. Figure 8 shows the evolution of the relative permittivity (a) and the loss tangent (b) as a function of frequency from 0.3 to $2 \mathrm{THz}$. Markers indicate again the average values over three samples fabricated under same conditions and color shade backgrounds are the standard deviations over the three samples. Experimental points corresponding to the $\mathrm{Al}_{2} \mathrm{O}_{3}$ sample composed of $d_{50}=1 \mu \mathrm{m}$ grain size and $0 \%$ porosity (in blue), already plotted in Fig. 8, have been plotted again for comparison with those corresponding to the above-described alumina sample with the zirconium dioxide impurity. For the sample with zirconium, the permittivity slightly increases as a function of $\mathrm{THz}$ frequency, as shown in Fig. 12(a). We also note that the standard deviation is larger, specially for frequencies lower than $0.7 \mathrm{THz}$. Again, the dielectric loss tangent increases as a function of $\mathrm{THz}$ frequency (Fig. 12(b)). 

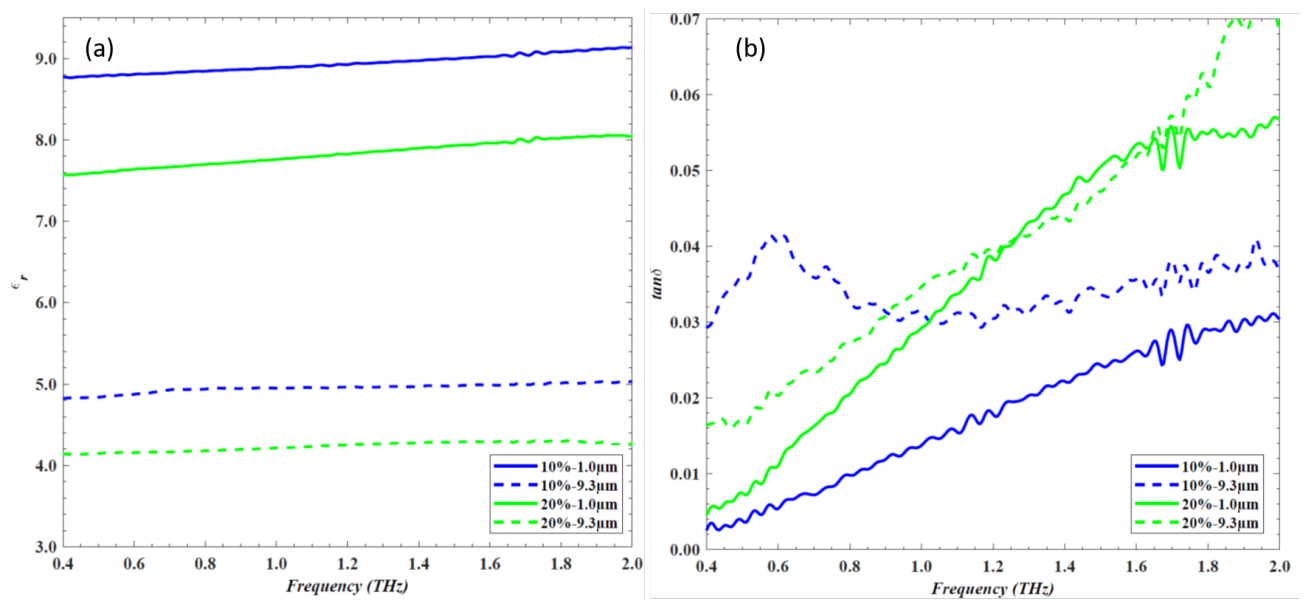

Figure 11: Dielectric properties of $\mathrm{Al}_{2} \mathrm{O}_{3}$ samples composed of $1 \mu \mathrm{m} d_{50}$ grain size and $9.3 \mu \mathrm{m} d_{50}$ grain size, with porosity fractions equal to $0 \%$ (yellow markers), $10 \%$ (blue markers) and 20\% (red markers). (a) Permittivity. (b) Loss tangent. Markers correspond to the average values measured over three different samples.
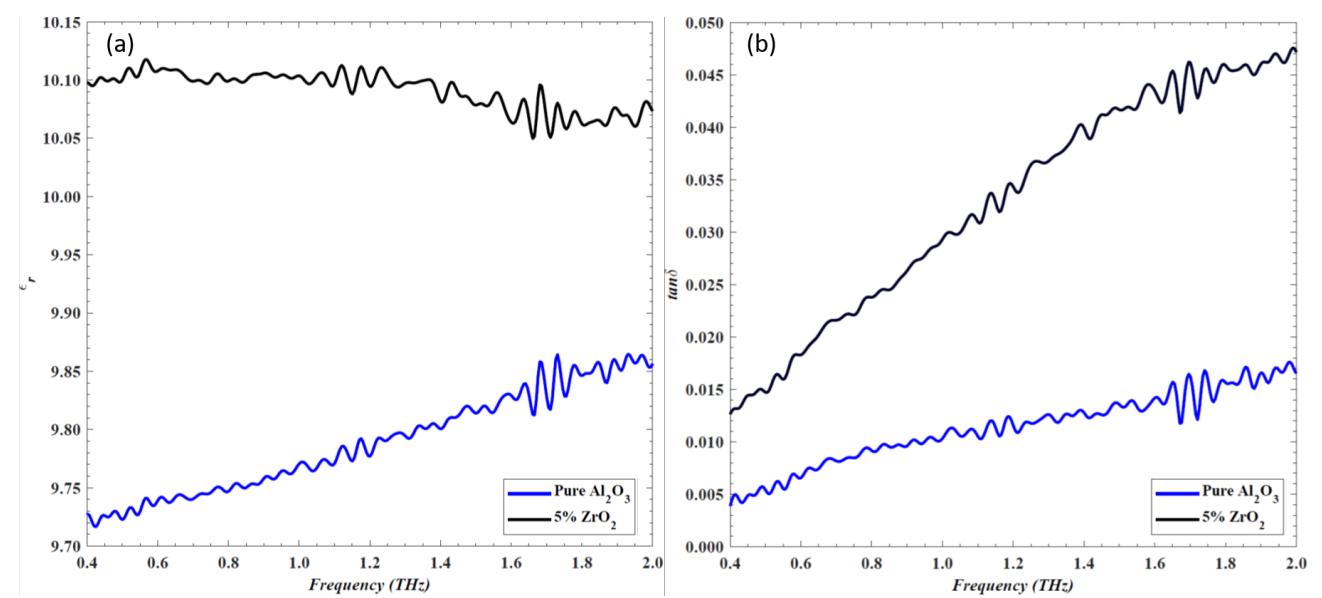

Figure 12: Dielectric properties of: (blue) $\mathrm{Al}_{2} \mathrm{O}_{3}$ sample composed of $1 \mu \mathrm{m} d_{50}$ grain size and $0 \%$ porosity ; (red) $\mathrm{Al}_{2} \mathrm{O}_{3}$ sample composed of $1 \mu \mathrm{m} d_{50}$ grain size with $5 \%$ vol $\mathrm{ZrO}_{2}$ impurities. (a) Relative permittivity. (b) Loss tangent. Markers correspond to the average values measured over three different samples.

We also compare these results with the dielectric properties of the $\mathrm{Al}_{2} \mathrm{O}_{3}$ sample composed of $d_{50}=1 \mu \mathrm{m}$ grain size and $0 \%$ porosity. Clearly, for all considered $\mathrm{THz}$ frequencies, the permittivity is higher for the sample with impurities. This may be attributed to the larger density of the sample with zirconium inclusions compared to the pure $\mathrm{Al}_{2} \mathrm{O}_{3}$ sample. We observe a similar behavior for the dielectric loss tangent, which is larger for the sample with the zirconium impurity. This additional experiment shows that THz time-domain spectroscopy is also a convenient and powerful method to detect impurities embedded in ceramic materials. Considering that the uncertainty of relative permittivity measurement is around 0.1 at $1 \mathrm{THz}$, we can estimate that the lowest $\mathrm{ZrO}_{2}$ impurities concentration that can be detected is around $1 \%$.

\section{Conclusion}

We reported on the non-destructive and contactless evaluation of $\mathrm{Al}_{2} \mathrm{O}_{3}$ ceramic porosity using terahertz time-domain spectroscopy. Up to $2 \mathrm{THz}$, the extraction of the relative permittivity and dielectric loss tangent of different porous samples and the help of an effective medium approximation make it possible to reveal a simple linear relationship between the permittivity and the sample porosity 
within the $0 \%$ to the $20 \%$ fraction range. From a given set of samples with a calibrated fabrication process, this can be applied to non-destructively determine the porosity of an unknown sample. In addition, we tested our approach by introducing changes in the fabrication parameters of the alumina ceramic, stimulating both structural and material modifications. Our results indicated that $\mathrm{THz}$ time-domain spectroscopy can easily differentiate alumina samples fabricated with different grain sizes or with impurity inclusions. More generally, these results demonstrate the potential of $\mathrm{THz}$ radiation for the non-destructive analysis of ceramic materials, providing an alternative evaluation technique for the fabrication and quality maintenance of ceramic products. Besides, we also pointed out that, owing to their high $\mathrm{THz}$ permittivity and low losses, ceramic materials could provide a new class of optical components and encourage the development of $\mathrm{THz}$ photonics.

\section{Declaration of Competing Interest}

The authors declare no known competing financial interests or personal relationships influencing the presented work.

\section{Acknowledgment}

This work is financed by the Région NouvelleAquitaine and the i2s company. The authors want to thanks all the EXALYSE $E_{b y C T T C}$ team for the SEM and mercury porosimetry characterizations and technical support (exalyses@cttc.fr).

\section{References}

[1] W. L. Chan, J. Deibel, D. M. Mittleman, Imaging with terahertz radiation, Reports on Progress in Physics 70 (8) (2007) 1325-1379. doi: $10.1088 / 0034-4885 / 70 / 8 / R 02$.

URL http://stacks.iop.org/ 0034 $-4885 / 70 / i=8 / a=R 02$ ?key=crossref . $62 \mathrm{dbc} 43 \mathrm{c} 846162263 \mathrm{df} 14 \mathrm{f} 25 \mathrm{f} 0 \mathrm{c} 9 \mathrm{bf} 17$

[2] P. H. Bolivar, M. Brucherseifer, J. G. Rivas, R. Gonzalo, I. Ederra, A. L. Reynolds, M. Holker, P. d. Maagt, Measurement of the dielectric constant and loss tangent of high dielectric-constant materials at terahertz frequencies, IEEE T Microw Theory 51 (2003). doi: 10.1109/TMTT. 2003.809693.

[3] K. Berdel, J. G. Rivas, P. H. Bolivar, P. d. Maagt, H. Kurz, Temperature dependence of the permittivity and loss tangent of high-permittivity materials at terahertz frequencies, IEEE Trans. Comp. Packag. Man. 53 (4) (2005) 1266-1271. doi:10.1109/TMTT.2005. 845752 .
[4] K. Z. Rajab, M. Naftaly, E. H. Linfield, J. C. Nino, D. Arenas, D. Tanner, R. Mittra, M. Lanagan, Broadband Dielectric Characterization of Aluminum Oxide (Al2O3), J. Micr. El. Pack. 5 (1) (2008) 2-7. doi: 10.4071/1551-4897-5.1.1.

[5] J. A. Hejase, P. R. Paladhi, P. P. Chahal, Terahertz characterization of dielectric substrates for component design and nondestructive evaluation of packages, IEEE Trans. Comp. Packag. Man. 1 (11) (2011) 1685-1694. doi: 10.1109/TCPMT . 2011.2163632.

[6] A. K. Klein, J. Hammler, C. Balocco, A. J. Gallant, Machinable ceramic for high performance and compact $\mathrm{THz}$ optical components, Opt. Mater. Express 8 (7) (2018) 1968-1975. doi:10.1364/OME.8.001968.

[7] M. Ma, Y. Wang, M. Navarro-Cía, F. Liu, F. Zhang, Z. Liu, Y. Li, S. M. Hanham, Z. Hao, The dielectric properties of some ceramic substrate materials at terahertz frequencies, J. Eur. Ceram. Soc. 39 (14) (2019) 4424 - 4428. doi:https://doi.org/10.1016/j. jeurceramsoc.2019.06.012.

[8] Y. Wada, Y. Urata, H. Ito, Y. Higashi, Measuring material parameters using a tunable monochromatic terahertz wave source, Appl. Opt. 59 (32) (2020) 10035. doi:10.1364/A0.406019.

[9] M. Zhai, A. Locquet, M. Jung, D. Woo, D. S. Citrin, Characterization of nanoporous $\mathrm{Al}_{2} \mathrm{O}_{3}$ films at terahertz frequencies, Opt. Lett. 45 (14) (2020) 4092-4095. doi : 10.1364/OL . 390129.

[10] M. Naftaly, J. Leist, R. Dudley, Investigation of ceramic boron nitride by terahertz time-domain spectroscopy, J. Eur. Ceram. Soc. 30 (12) (2010) 2691-2697. doi: 10.1016/j.jeurceramsoc.2010.04.040.

[11] B. Synkiewicz-Musialska, D. Szwagierczak, J. Kulawik, N. Pałka, P. Bajurko, Impact of additives and processing on microstructure and dielectric properties of willemite ceramics for LTCC terahertz applications, J. Eur. Ceram. Soc. 40 (2) (2020) 362-370. doi:10.1016/ j.jeurceramsoc.2019.10.005.

[12] D. Wu, C. Haude, R. Burger, O. Peters, Application of terahertz time domain spectroscopy for NDT of oxide-oxide ceramic matrix composites, Infrared Phys. Technol. 102 (2019) 102995. doi:10.1016/j.infrared. 2019.102995.

[13] [link]. URL https : //www.iso.org/standard/69745.html

[14] [link]. URL https: //www . iso.org/standard/56005.html

[15] G. Kent, Nondestructive permittivity measurement of substrates, IEEE Trans. Instrum. Meas. 45 (1) (1996) 102-106. doi:10.1109/19.481319.

[16] D. Ye, W. Wang, H. Zhou, J. Huang, W. Wu, H. Gong, Z. Li, In-situ evaluation of porosity in thermal barrier coatings based on the broadening of terahertz timedomain pulses: simulation and experimental investigations, Opt. Express 27 (20) (2019) 28150-28165. doi: 10.1364/OE. 27.028150 .

[17] J. Neu, C. Schmuttenmaer, Tutorial: An introduction to terahertz time domain spectroscopy (thz-tds), Journal of Applied Physics 124 (2018) 231101. doi: 10.1063/1.5047659.

[18] T. C. Choy, Effective Medium Theory: Principles and Applications, Oxford University Press, 2016, googleBooks-ID: hS8iCwAAQBAJ.

[19] M. Hamdi, F. Garet, L. Duvillaret, P. Martinez, G. E. P. Tourtollet, Identification tag in the terahertz 
frequency domain using low-cost and tunable refractive index materials, annals of telecommunications - annales des télécommunications 68 (7-8) (2013) 415-424. doi:10.1007/s12243-013-0374-7.

URL http://link.springer.com/article/10.1007/ s12243-013-0374-7

[20] X. Lu, H. Sun, T. Chang, J. Zhang, H. Cui, Terahertz detection of porosity and porous microstructure in pharmaceutical tablets: A review, International Journal of Pharmaceutics 591 (2020) 120006. doi:10.1016/j.ijpharm.2020.120006.

URL https://linkinghub.elsevier.com/retrieve/ pii/S0378517320309911

[21] J. C. M. Garnett, J. Larmor, XII. Colours in metal glasses and in metallic films, Philosophical Transactions of the Royal Society of London. Series A, Containing Papers of a Mathematical or Physical Character 203 (359-371) (1904) 385-420, publisher: Royal Society. doi:10.1098/rsta.1904.0024.

URL https://royalsocietypublishing.org/doi/10. 1098/rsta.1904.0024

[22] S. O. Nelson, T.-S. You, Relationships between microwave permittivities of solid and pulverised plastics, Journal of Physics D: Applied Physics 23 (3) (1990) 346-353, publisher: IOP Publishing. doi:10.1088/ 0022-3727/23/3/014.

URL https://doi.org/10.1088/0022-3727/23/3/014

[23] H. Looyenga, Dielectric constants of heterogeneous mixtures, Physica 31 (3) (1965) 401-406. doi : 10.1016/0031-8914(65)90045-5.

URL http://www.sciencedirect.com/science/ article/pii/0031891465900455

[24] H. Fricke, A Mathematical Treatment of the Electric Conductivity and Capacity of Disperse Systems I. The Electric Conductivity of a Suspension of Homogeneous Spheroids, Physical Review 24 (5) (1924) 575-587. doi:10.1103/PhysRev.24.575.

URL https://link.aps.org/doi/10.1103/PhysRev. 24.575

[25] V. I. Kushch, R. Springhetti, S. V. Shmegera, Effective permittivity of composite elastomer with account of electric conductivity of phases and imperfect interface, International Journal of Engineering Science 123 (2018) 51-61. doi:10.1016/j.ijengsci.2017.11.016. URL https://www.sciencedirect.com/science/ article/pii/S0020722517322875

[26] M. Scheller, C. Jansen, a. M. Koch, Applications of Effective Medium Theories in the Terahertz Regime, Recent Optical and Photonic TechnologiesPublisher: IntechOpen (Jan. 2010). doi:10.5772/6915.

URL https://www.intechopen.com/books/ recent-optical-and-photonic-technologies/

applications-of-effective-medium-theories-in-the-terahertz-regime

[27] D. a. G. Bruggeman, Berechnung verschiedener physikalischer Konstanten von heterogenen Substanzen. I. Dielektrizitätskonstanten und Leitfähigkeiten der Mischkörper aus isotropen Substanzen, Annalen der Physik 416 (7) (1935) 636-664, _eprint: https://onlinelibrary.wiley.com/doi/pdf/10.1002/andp.19354160705. doi : https://doi.org/10.1002/andp.19354160705. URL https://onlinelibrary.wiley.com/doi/abs/10. 1002/andp. 19354160705

[28] J. E. Spanier, I. P. Herman, Use of hybrid phenomenological and statistical effective-medium theories of dielectric functions to model the infrared reflectance of porous SiC films, Physical Review B 61 (15) (2000) 10437-10450, publisher: American Physical Society. doi: 10.1103/PhysRevB.61.10437.

URL https://link.aps.org/doi/10.1103/PhysRevB. 61.10437

[29] D. Markl, P. Wang, C. Ridgway, A.-P. Karttunen, M. Chakraborty, P. Bawuah, P. Pääkkönen, P. Gane, J. Ketolainen, K.-E. Peiponen, J. A. Zeitler, Characterization of the Pore Structure of Functionalized Calcium Carbonate Tablets by Terahertz Time-Domain Spectroscopy and X-Ray Computed Microtomography, Journal of Pharmaceutical Sciences 106 (6) (2017) 1586-1595. doi:10.1016/j.xphs.2017.02.028.

URL https://www.sciencedirect.com/science/ article/pii/S0022354917301430

[30] A. Brovelli, G. Cassiani, Effective permittivity of porous media: a critical analysis of the complex refractive index model, Geophysical Prospecting 56 (5) (2008) 715-727, _eprint: https://onlinelibrary.wiley.com/doi/pdf/10.1111/j.13652478.2008.00724.x. doi:https://doi.org/10.1111/j. 1365-2478.2008.00724.x.

URL https://onlinelibrary.wiley.com/doi/abs/10. 1111/j.1365-2478.2008.00724.x

[31] S. B. Jones, S. P. Friedman, Particle shape effects on the effective permittivity of anisotropic or isotropic media consisting of aligned or randomly oriented ellipsoidal particles, Water Resources Research 36 (10) (2000) 2821-2833, _eprint: https://agupubs.onlinelibrary.wiley.com/doi/pdf/10.1029/2000WR90019 doi:https://doi.org/10.1029/2000WR900198.

URL https://agupubs.onlinelibrary.wiley.com/ doi/abs/10.1029/2000WR900198

[32] P. Bawuah, N. Tan, S. N. A. Tweneboah, T. Ervasti, J. Axel Zeitler, J. Ketolainen, K.-E. Peiponen, Terahertz study on porosity and mass fraction of active pharmaceutical ingredient of pharmaceutical tablets, European Journal of Pharmaceutics and Biopharmaceutics 105 (2016) 122-133. doi:10.1016/j.ejpb.2016.06.007.

URL https://www.sciencedirect.com/science/ article/pii/S0939641116302144

[33] W. J. Tropf, M. E. Thomas, - Aluminum Oxide (Al2O3) Revisited, in: E. D. Palik (Ed.), Handbook of Optical Constants of Solids, Academic Press, Burlington, 1997, pp. 653-682. doi : 10.1016/B978-012544415-6.50124-2.

URL https://www.sciencedirect.com/science/ article/pii/B9780125444156501242 